\title{
ESTUDIO CUALITATIVO DE LAS MOTIVACIONES DEL ALUMNADO DE BACHILLERATO EN REFERENCIA A LA MODALIDAD DE ESTUDIOS.
}

\author{
QUALITATIVE STUDY OF THE STUDENT'S MOTIVATIONS OF HIGH SCHOOL \\ REGARDING THE MODALITY OF STUDIES.
}

\author{
Mario Corrales Serrano*, José Moreno Losada*, Jesús Sánchez Martín*, \\ Francisco Zamora Polo** \\ Universidad de Extremadura*. Universidad de Sevilla**
}

\begin{abstract}
Correspondencia: Mario Corrales Serrano
Correo: mariocorralesserrano@gmail.com
\end{abstract}

Recibido: 23/04/2019; Aceptado: 19/11/2019

DOI: 10.17398/0213-9529.39.1.85

\begin{abstract}
Resumen
El presente trabajo es un acercamiento a las motivaciones intrínsecas y extrínsecas que llevan al alumnado de Bachillerato a decantarse por una modalidad de estudios en concreto. Desde el ámbito de la Didáctica de las Ciencias Sociales resulta relevante conocer estas motivaciones, para poder diseñar estrategias didácticas que mejoren el proceso de enseñanza y aprendizaje, haciéndolo más motivador. El procedimiento seguido en el presente estudio ha consistido en analizar escritos de alumnos de todas las modalidades de Bachillerato un colegio de la provincia de Badajoz, en los que respondían a cuestiones acerca de los motivos que los habían llevado a decantarse por su modalidad de estudios. Para llevar a cabo el análisis se ha utilizado una metodología cualitativa, que nos permite poder valorar de manera suficientemente rica los factores que influyen en la motivación de las decisiones del alumnado. Los resultados apuntan a que el alumnado de la modalidad de Ciencias está más motivado extrínsecamente que el alumnado de Humanidades y Ciencias Sociales, lo que señala la necesidad de trabajar factores de motivación extrínseca en estas modalidades.
\end{abstract}

Palabras claves: Motivación; Metodología cualitativa; Bachillerato; Educación Secundaria.

\begin{abstract}
The present paper is an approach to the intrinsic and extrinsic motivations that lead the students of High School to opt for a specific study modality. From the scope of the Social Sciences Didactics it is relevant to know these motivations, to be able to design didactic strategies that improve the teaching and learning process, making it more motivating. The procedure followed in the present study consisted of analysing the writings of students of all the High School modalities of a school in the province of Badajoz, in which they answered questions about the reasons that had led them to opt for their studies. To carry out the analysis, a qualitative methodology has been used, which allows us to assess in a sufficiently rich way the factors that influence the motivation of student decisions. The results suggest that the students of the Sciences modality are more extrinsically motivated than the students of Humanities and Social Sciences, which indicates the need to work with extrinsic motivation factors in these modalities.
\end{abstract}

Key Words: motivation; qualitative methodology; High school; Secondary Education.

Campo Abierto, v. 39, n. 1, p. 85-99, 2020 


\section{INTRODUCCIÓN}

Una de las decisiones más complicadas que un alumno de Educación Secundaria Obligatoria (ESO) debe tomar a lo largo de esta etapa se centra en la modalidad de estudios que desea cursar. Aunque en los últimos cursos de la ESO el alumnado va tomando una serie de opciones que le orientan en su itinerario educativo, es la modalidad del Bachillerato la que orienta de manera definitiva al alumnado hacía unos estudios concretos.

Este estudio tiene como finalidad principal conocer los elementos que determinan o que influyen en la decisión del alumnado de Bachillerato de decantarse por una modalidad de estudios concreta, en detrimento de las demás opciones. Para poder plantear estrategias didácticas innovadoras para motivar al alumnado de Ciencias Sociales de cara al aprendizaje de estas materias resulta útil conocer los factores que hacen que el alumnado se decante por las diversas modalidades.

Partimos de la base de que hay un reparto de alumnado un tanto desigual entre las distintas modalidades (como expondremos más adelante con datos concretos), y también valoramos la posibilidad de que influyan en la decisión elementos motivacionales de tipo intrínseco y extrínseco.

Nuestro trabajo se lleva a cabo desde el marco didáctico del área de las Humanidades y las Ciencias Sociales, desde el presupuesto teórico de que si conocemos los factores que motivan al alumnado para inclinarse por una modalidad concreta de estudios, podemos incidir en el desarrollo didáctico de las materias, para mejorar la motivación.

\subsection{Estructura del Bachillerato actual}

El actual sistema educativo ofrece al estudiante de Bachillerato tres modalidades de estudio, una de ellas con dos itinerarios, desde las que se puede acceder posteriormente a diferentes estudios universitarios, dependiendo de un sistema de ponderación.

La tabla 1 muestra esta estructura básica:

Tabla 1:

Estructura de Bachillerato.

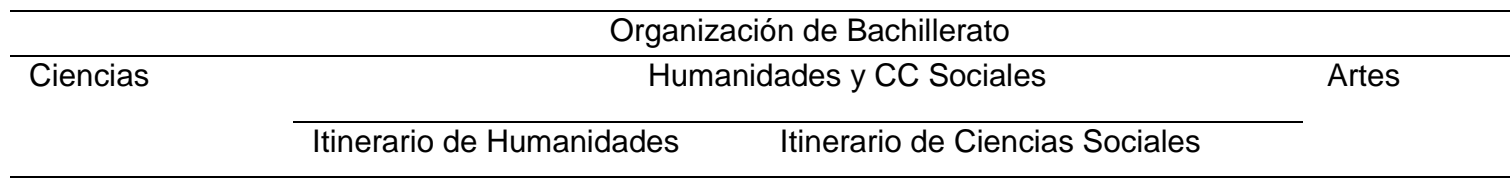

Fuente: https://www.mecd.gob.es/educacion-mecd/areas-educacion/estudiantes/bachillerato.html

Basándose en esta misma división, las pruebas de evaluación de Bachillerato para el acceso a la universidad (EBAU) están diseñadas en dos fases, acceso y admisión, en las que tiene un peso determinado la asignatura troncal de modalidad y las de opción. De esta manera, en el curso de $2^{\circ}$ de Bachillerato, por ejemplo, el alumnado debe cursar obligatoriamente las asignaturas de Historia de España, Lengua y Literatura e Idioma extranjero, mientras que las demás asignaturas dependen de la modalidad de estudios escogida, itinerario, y materias "optativas" de cada modalidad. Son más, por tanto, las asignaturas optativas que las obligatorias para todo el conjunto del alumnado, y la carga motivacional desde la que se 
enfrenta el alumnado a esta elección no sólo determinará su éxito académico en la etapa, sino que orientará sus posibilidades de acceso a los diferentes grados universitarios.

Teniendo todo esto en cuenta, resulta de gran importancia profundizar es los factores motivacionales del alumnado para esta etapa concreta, y en su posible influencia en las didácticas específicas de las materias.

\subsection{La motivación: definición y aplicación del concepto a nuestro trabajo}

El constructo sobre el que se desarrolla el estudio empírico de este trabajo es la motivación. Como punto de partida debemos definir qué entendemos por motivación, llegando a las raíces teóricas del concepto, y cuáles son nuestras referencias principales en el ámbito de la psicología de la educación y en la didáctica.

La primera referencia para hablar del concepto de motivación es su origen etimológico en el verbo latino movere, que se puede traducir como "moverse, estar en movimiento", o teniendo en cuenta un punto de vista más dinámico, estar listo para emprender un movimiento o una acción. Los parámetros en los que se desarrolla esta definición se sitúan en un doble origen de la motivación, que va a ser muy interesante para nuestro trabajo:

- Por un lado, una disposición interna en relación con una cuestión.

- Por otro lado, la capacidad de reaccionar externamente, ante una necesidad, un estímulo o una meta.

La definición que ofrece el diccionario de la (RAE, 2014) cuando incide en las mismas ideas que acabamos de exponer:

1. Acción y efecto de motivar.

2. Motivo (causa).

3. Conjunto de factores internos o externos que determinan en parte las

Acciones de una persona."

La tercera acepción que recoge el diccionario de la RAE nos pone de nuevo en la pista de un concepto relacionado con la acción y con las causas que provocan dicha acción en la persona (Huertas, 1997).

Entrando en el ámbito de la psicología educativa encontramos algunos rasgos que concretan nuestra idea inicial de motivación. Puede servir como punto de partida la siguiente definición:

"La motivación es el proceso que trata de explicar cómo el conjunto de pensamientos, creencias y emociones se transforman en una acción determinada a la consecución de un objetivo.

Es el proceso por el cual la actividad dirigida a un fin es instigada y mantenida." (Pintrich, Schunk, y Luque, 2006).

Tres son las dimensiones de la motivación que apreciamos en esta definición, y que pueden ser interesantes para nuestro trabajo: una dimensión activadora, que pone en movimiento a la persona; una dimensión directiva, que señala el objetivo hacia el que se dirige dicho movimiento, y una dimensión persistente, que otorga a la persona el empuje necesario para no desistir hasta no haber conseguido el objetivo que se plantea (Mallart, 2008). Teniendo en cuenta estas tres dimensiones de la motivación podemos resaltar que la motivación implica una disposición interior, un objetivo que se pretende conseguir, y un motor que no sólo sirva de arranque, sino que mantenga el impulso hasta lograr el objetivo planteado (Queija et al., 2014). 
A lo largo de la historia reciente de la psicología son muchas las teorías psicológicas desde las que se ha explicado esto que entendemos por motivación. En la tabla 2 presentamos resumidamente las teorías clásicas a este respecto, para que sirva de referencia teórica histórica de la reflexión del constructo motivación, fundamental en este trabajo.

Tabla 2:

Principales teorías de la motivación. Fuente: Elaboración propia.

\section{Principales teorías de la motivación}

\begin{tabular}{|c|c|c|}
\hline Autor & Nombre & Descripción \\
\hline (Maslow, 1991) & Necesidades & $\begin{array}{l}\text { Las motivaciones nacen de las necesidades de la } \\
\text { persona. Según la clasificación de necesidades, así } \\
\text { necesita de las motivaciones. }\end{array}$ \\
\hline (Atkinson, 1983) & Logro & $\begin{array}{l}\text { La conducta humana se encamina a la consecución } \\
\text { de logros de objetivos, para los que se necesita } \\
\text { motivación. }\end{array}$ \\
\hline (Weiner, 1985) & Atribución & $\begin{array}{l}\text { El ser humano atribuye a factores internos o } \\
\text { externos todo lo que sucede. Esto es un factor clave } \\
\text { en la motivación. }\end{array}$ \\
\hline (Bandura, 1986) & Aprendizaje social & $\begin{array}{l}\text { Las creencias, expectativas, intenciones, metas y } \\
\text { valores son elementos que influyen de un modo } \\
\text { determinante para explicar la motivación en el } \\
\text { proceso cognitivo. }\end{array}$ \\
\hline
\end{tabular}

Estas definiciones clásicas de motivación permiten interpretarla como el motor de toda acción humana, que pone todos los recursos de la persona en disposición de alcanzar un objetivo. Es necesario tenerlas en cuenta para este trabajo, ya que en ellas se apoyan los matices de los planteamientos motivacionales más recientes, que se introducen a continuación.

Desde el plano de la psicología moderna se han enriquecido estas teorías, con la puesta en valor de factores, como las expectativas del profesor sobre el alumnado, la autostima, la autoeficacia y el autoconcepto (Queija et al., 2014).

Como complemento de todo lo expuesto hasta el momento para entender adecuadamente el proceso por el que se pone en marcha este motor hemos de hablar, como (Marina, 2013) de los mecanismos de la motivación, es decir, de la estructura psicológica y antropológica que hace posible que la persona se movilice en dirección a un objetivo, y a su vez, sea capaz de mantener la intensidad del esfuerzo para lograr alcanzar el objetivo. Así, (Marina, 2013) distingue entre dos momentos del fenómeno:

- Motivación inicial, que está presente en la toma de decisión de dirigir la acción de la persona hacia un objetivo concreto y hace que todo se ponga en marcha.

- Motivación para la tarea, que acompaña a la persona en todo el proceso de consecución del objetivo y que le sirve de fuerza motora para poder lograrlo a pesar de las dificultades que puedan presentarse.

Es doble distinción es reforzada con las teorías motivacionales recientes, basadas en la neuroeducación, que remarcan ese proceso psicológico en relación con el proceso de acciónrecompensa (Bacigalupe y Mancini, 2012).

Como conclusión a este acercamiento teórico a la motivación desde el plano de la psicología y de la educación podemos afirmar que la motivación es un proceso, es decir, un conjunto de elementos que están interrelacionados de forma compleja, y que, si se activan de 
modo adecuado, tienen como resultado la consecución de un objetivo. A su vez, es importante resaltar que al hablar de la motivación se habla de un proceso que hace que la persona se ponga en movimiento para conseguir un objetivo por el que se siente atraído y que desea lograr, y que acompaña todo el proceso, sirviendo de fuerza impulsora para la consecución de dicho objetivo.

Para esta investigación resulta importante situarse de entrada ante esta idea procesual, ya que nos introduce en el contexto adecuado desde el que podemos apreciar los diferentes factores que influyen en el fenómeno complejo de la motivación, así como la relación que existe entre dichos factores. En la aplicación práctica del concepto motivación que se va a llevar a cabo en la elección de modalidad del alumnado de Bachillerato se ha de observar este proceso, con elementos diferentes que posibilitan la decisión final del alumnado. Si el objetivo de este trabajo es profundizar en el modo en el que la motivación influye en el proceso de enseñanza y aprendizaje, para poder usarla en la estrategia didáctica, resulta realmente interesante esta visión, ya que nos presenta la motivación como el motor que puede hacer que el alumnado se mueva para la consecución de un objetivo académico, siempre que ese objetivo sea objeto de deseo para la persona.

\subsection{Motivación intrínseca y extrínseca}

\subsubsection{Punto de vista psicológico del ciclo de la motivación}

Hasta ahora se ha abordado la definición del fenómeno de la motivación, desde los parámetros de la etimología del término, y desde las primeras y más sencillas aportaciones de la psicología. Después de todo lo que se ha presentado como punto de partida acerca del proceso de la motivación, hay un elemento clave por el que nos debemos preguntar en este momento de la reflexión: ¿cuál es el origen que hace que se ponga en marcha el proceso de la motivación? ¿De qué tipo son los resortes que se ponen en funcionamiento para que podamos decir que una persona está o no motivada para alcanzar un objetivo?

A lo largo del recorrido teórico que venimos exponiendo hemos hablado de que el proceso de la motivación está relacionado con el deseo que una persona siente en relación con la consecución de un objetivo concreto, pero ¿qué hace que se desee conseguir un objetivo, y sin embargo no se desee conseguir otro? ¿Por qué un objetivo resulta atrayente para unas personas y para otras no?

Sin duda es difícil dar respuesta a estas preguntas. Para buscar una posible respuesta, podemos adentrarnos en el campo de todo lo que pone en relación la psicología de la persona, y con la acción de la persona (antropología práctica/ética). En esta misma dirección, es necesario apuntar un camino para responder a estas cuestiones desde la ética de la felicidad, (Cortina, 2012; Hortal, 2002), que pone encima de la mesa los motivos por los que una persona se dirige a unos objetivos concretos.

En un primer momento, es necesario hablar de dos tipos de origen en esta motivación:

- Motivación intrínseca es la motivación cuyo punto de arranque está en el interés que el objetivo despierta en sí mismo, por el hecho de ser lo que es.

- Motivación extrínseca, que hace referencia a un motor que está fuera del objetivo en sí, y que se deriva de la consecución del objetivo (Mallart, 2008).

Estos dos tipos de motivación nos sitúan ante un escenario de respuesta a la cuestión de por qué se motivan los alumnos en el estudio con una doble posibilidad: en algunas ocasiones, la motivación puede ser el fruto de la búsqueda de sentirse bien con uno mismo, cumplir con los propios objetivos o satisfacer el placer de aprender, mientras que, en otras ocasiones, se 
puede buscar lograr metas que no tienen que ver directamente con el contenido de lo que se estudia, como por ejemplo, la consecución de un premio tras unos buenos resultados académicos.

Aplicando este desarrollo teórico a la cuestión de la motivación en la elección de estudios, se podría entender que un alumno está motivado intrínsecamente para estudiar una modalidad de estudios cuando siente vocación y le gustan los contenidos de esas materias, mientras que actuaría la motivación extrínseca cuando la elección se ve determinada por la búsqueda de una determinada posición social o económica.

Atendiendo a lo expuesto anteriormente, los factores externos de motivación pueden ser propiciados desde el exterior de la persona, mientras que los internos tienen que ver con la personalidad, los gustos o la vocación. Siendo esto así, ¿es posible incidir en la motivación de los estudiantes? El desarrollo teórico que se ha expuesto anteriormente nos presentaba el concepto de motivación como un concepto dinámico, de manera que ambos tipos de motivación pueden retroalimentarse positiva o negativamente. Este carácter continuo del ciclo de la motivación se puede ver afectado por algunos factores, entre los que destaca la actuación del profesor, y la posibilidad de conseguir éxito en la tarea propuesta (Woolfolk, 2006).

\subsubsection{Un punto de vista ético de la cuestión de las motivaciones}

Desde el punto de vista de la búsqueda de una formación integral para los estudiantes, es importante para los objetivos de esta investigación introducir la variable de la relación que existe entre los elementos que motivan a una persona en su proceso cognitivo y a construcción de la propia persona, por varios motivos.

En primer lugar, partimos de la afirmación de que toda acción humana está afectada por cierta disposición ética, (Moreno, Sanchez y Zamora, 2014; Moreno, 2015) de manera que los elementos motivadores de los que venimos hablando en este trabajo no permanecen en un terreno neutral, sino que forman parte de la persona, de su visión de la vida, de su ética.

En este sentido, hay que recordar que el concepto motivación está directamente relacionado con la consecución de un objetivo. En el caso de la motivación en el proceso cognitivo, así como en la elección del itinerario educativo, parece tener claramente el horizonte de la vida profesional: Los estudiantes que eligen una u otra opción en Bachillerato se están orientando en función de su deseo por acceder a unos estudios universitarios concretos. Los estudiantes universitarios, a su vez, se preparan para desempeñar una profesión. Así pues, en la elección de dicha profesión están implicados elementos motivacionales internos y externos que tienen que ver con la consecución de un objetivo concreto: la autorrealización de la persona. En este plano, podemos poner en paralelo la visión intrínseca y extrínseca de la motivación con el planteamiento ético de los bienes internos y externos (Moreno; Sanchez, y Zamora, 2014) del objetivo que una persona quiere alcanzar en un proceso concreto, como puede ser la elección de una modalidad de estudios concreta.

\section{METODOLOGÍA}

\subsection{Objetivos de la investigación}

El análisis teórico acerca de la motivación y sus implicaciones permite plantear una investigación acerca de cómo afecta a la decisión de optar por una modalidad concreta de estudios en el alumnado de Bachillerato buscando los siguientes objetivos:

1. Analizar si es posible detectar motivaciones concretas en el relato de alumnos de Bachillerato sobre su elección de modalidad de estudios. 
2. Distinguir elementos de tipo intrínseco y extrínseco en las posibles motivaciones que formule el alumnado de Bachillerato sobre su elección de modalidad.

3. Establecer distinciones de motivaciones de tipo intrínseco y extrínseco en relación con el alumnado que opta por la modalidad de Ciencias Sociales frente al alumnado de otras modalidades. Este tercer objetivo es el que permitirá señalar líneas de orientación para la implementación de estrategias motivadoras para los estudiantes de Bachillerato.

\subsection{Descripción de la muestra}

Las características del estudio que nos ocupa exigen la selección de una muestra que se adecúe principalmente a dos condiciones necesarias:

- Estar cursando estudios de Educación Secundaria en la etapa de Bachillerato.

- Haberse definido por una de las posibles modalidades por las que se puede optar en esta etapa de estudios.

Por este motivo, hemos optado por un muestreo de conveniencia, eligiendo a sujetos que cumplieran con las condiciones establecidas. El total de la muestra es de 22 sujetos, chicos y chicas pertenecientes a los dos cursos de la etapa de Bachillerato, de un colegio concertado de la provincia de Badajoz. Algunas características interesantes de la muestra son las siguientes:

a) Edad: Los sujetos de la muestra tienen edades comprendidas entre los 16 y los 20 años, que se corresponden con las edades mínimas de inicio de los estudios de la etapa de Bachillerato y con las edades máximas de repetidores de dichos cursos.

b) Género: En lo que se refiere al género, encontramos una distribución bastante equitativa ( $45 \%$ chicos y $55 \%$ chicas).

c) Situación socioeconómica: Según la zona de la ciudad en la que está situada el centro, y la categorización que hace la Junta de Extremadura de este, el alumnado de la muestra pertenece a familias de clase media y media-alta.

d) Modalidad de estudios: En relación con la modalidad de estudios tenemos una muestra que corresponde más o menos con los parámetros habituales en España, ya que hay un mayor porcentaje de alumnado que cursa Ciencias (60\%) que el resto de las modalidades (40\%).

La tabla 3 muestra la perspectiva de estudios de grado universitario de la muestra. Esta tabla tiene varias utilidades para nuestra investigación:

- Por una parte, el alumnado participante en la investigación tiene determinados sus objetivos, cosa que, como ya se ha expuesto, que es un elemento fundamental para adentrarnos en el análisis de las motivaciones.

- Por otro lado, nos encontramos con una amplitud y una variedad que puede hacer que nuestro análisis sea bastante rico, y conduzca a esta investigación a resultados satisfactorios.

- Por último, también interesa a este trabajo la observación de las titulaciones más demandadas, para poder asociarlas a las diferentes modalidades de estudio. 
Tabla 3:

Grados universitarios que elegiría el alumnado de nuestra muestra.

\begin{tabular}{lc}
\hline \multicolumn{1}{c}{ Listado de carreras universitarias deseadas } & Porcentaje de participantes (\%) \\
\hline Derecho & 18,75 \\
Educación & 15,62 \\
Fuerzas y cuerpos de seguridad del Estado & 12,5 \\
Ingeniería industrial & 9,35 \\
Física & 6,25 \\
ADE & 6,25 \\
Enfermería & 6,25 \\
Fisioterapia & 6,25 \\
Filosofía & 3,12 \\
Arquitectura & 3,12 \\
Ciencias del deporte & 3,12 \\
Diseño Gráfico & 3,12 \\
Turismo & 3,12 \\
Veterinaria & 3,12 \\
Ingeniería aeronáutica & 3,12 \\
Ingeniería agropecuaria & 3,12 \\
\hline
\end{tabular}

Para completar esta visión, es necesario tener en cuenta que, para parte de los participantes en esta muestra, sus preferencias de carrera aún no son definitivas, y están en función de diferentes variables que aparecerán a lo largo del análisis de resultados.

\subsection{Método e Instrumento de análisis}

El método que se ha utilizado para llevar a cabo este acercamiento de tipo cualitativo a la cuestión de las motivaciones del alumnado de Bachillerato en su elección de modalidad ha sido el análisis del contenido de una serie de textos elaborados por el alumnado de la muestra, respondiendo a nuestra petición de explicitar qué modalidad de estudios están cursando y por qué.

La propuesta concreta que le hemos hecho al alumnado es la elaboración de una redacción libre, más o menos de un folio de extensión, en la que tenían que responder a tres sencillas cuestiones:

- ¿Qué modalidad de estudios has elegido para el Bachillerato?

- ¿Qué carrera te gustaría estudiar?

- ¿ ¿Cuáles son los motivos que te llevan a decidirte por esa carrera?

En contraposición con la información que nos podría ofrecer un análisis de tipo cuantitativo, que nos mostraría una foto fija de la información que persigue este estudio, el análisis cualitativo nos ofrece algunas ventajas interesantes, que han hecho que se haya preferido trabajar con esta metodología para lograr los objetivos marcados:

- Por una parte, el alumnado se acerca a la cuestión propuesta sin las referencias concretas que le podrían ofrecen las preguntas de un cuestionario, con lo que la información sobre sus motivaciones que vamos a obtener no está determinada por las preguntas que le hacemos.

- $\quad$ Por otra parte, este formato de investigación permite al alumnado de la muestra expresar varios tipos de motivación, que no tendrían que ser excluyentes, y que nos pueden mostrar una visión complementaria.

Como es evidente, el análisis de las respuestas obtenidas y, por tanto, la identificación de ideas persistentes en el alumnado en relación con las motivaciones de elección de carrera se corresponde con una metodología cualitativa, entendida ésta como la investigación que produce datos descriptivos: palabras propias de personas (habladas o escritas) y la conducta 
observable (Quecedo y Castaño, 2002). En este sentido, Sandín define la investigación cualitativa del siguiente modo:

"La investigación cualitativa es una actividad sistemática orientada a la comprensión en profundidad de fenómenos educativos y sociales, a la transformación de las prácticas y los escenarios educativos, a la toma de decisiones y al descubrimiento de un cuerpo organizado de conocimientos" (Sandín, 2003, 123).

Por todo esto, consideramos oportuna esta metodología para llevar a cabo una investigación en el área de Ciencias Sociales (Ávila, Rivero y Domínguez, 2010).

Esta metodología no permite llegar a conclusiones universalizables, dado el número reducido de sujetos de la muestra, pero tiene algunas interesantes ventajas, como la posibilidad de analizar la visión de los sujetos estudiados sin que estén mediatizados por las preguntas de un cuestionario, o la posibilidad de dejar que se expresen según la inclinación de su pensamiento en relación con la cuestión que se les propone (Bisquerra, 2004)

Son muchos los estudios que avalan la fiabilidad de la investigación cualitativa en educación, algunos de ellos, citados anteriormente, que establecen los siguientes rasgos comunes (Eisner, 1998):

- Estudios centrados en contextos específicos.

- $\quad$ Los investigadores son el principal instrumento de medida de la investigación.

- Tienen naturaleza interpretativa.

- Se centran, como uno de sus elementos fundamentales, en el uso del lenguaje.

- Prestan especial atención a lo concreto.

La herramienta que hemos utilizado para analizar las redacciones libres de los participantes en esta parte de la investigación es el software de investigación cualitativa WebQDA, (Souza et al., 2016) que permite trabajar sobre las redacciones elaboradas por el alumnado de la muestra, de manera que se pueden asociar las ideas que aparecen en los textos a un sistema de nodos sobre los que se puede operar para sacar conclusiones globales de todos los textos. Utilizando esta herramienta, se ha llevado a cabo un proceso de análisis cualitativo de los textos, abriendo un proyecto, insertando las fuentes de estudio y aplicando diferentes procedimientos de análisis de contenido. El procedimiento de trabajo que se ha seguido con este software es el que se puede ver en la figura 1:
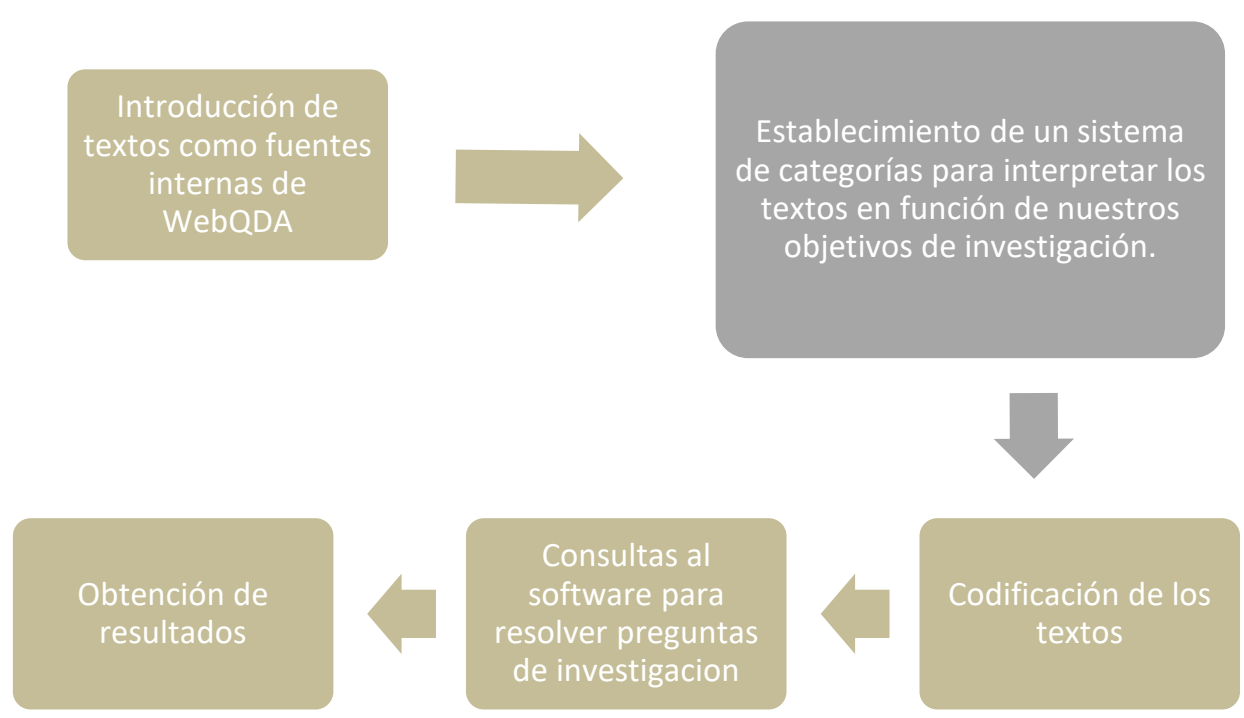

Figura 1: Procedimiento para crear proyectos de análisis cualitativo en WebQDA. Elaboración propia 
Siguiendo este modelo hemos llevado a cabo una investigación cualitativa siguiendo estos pasos:

1. En primer lugar, se ha solicitado al alumnado de nuestra muestra que elaboren una redacción en la que contesten de modo general a las tres cuestiones a las que hemos hecho referencia anteriormente. Para orientar su respuesta, hemos comenzado haciendo un recordatorio de la visita a la feria educativa organizada por la Universidad de Extremadura (UEx), en la que se les ha orientado acerca de sus posibilidades de acceso a diferentes titulaciones universitarias.

2. Una vez tenemos los textos en formato digital a nuestra disposición, se han abierto dos proyectos en el software de análisis cualitativo WebQDA, y se han introducido los textos como fuentes internas, de manera que pueden estar a nuestra disposición para ser analizados. En uno de los proyectos, se ha categorizado los textos del alumnado de la modalidad de Ciencias y en el otro con los pertenecientes a Humanidades y Ciencias Sociales, de manera que sea posible extraer conclusiones independientes de cada grupo y compararlas,

3. Para poder categorizar la información de los textos en función de los objetivos de la investigación, se ha establecido un sistema de nodos, siendo las categorías principales los dos tipos de motivación (intrínseca y extrínseca), y diversificando ambas posibilidades teniendo en cuenta criterios validados en cuestionarios de motivaciones empleados en otros estudios (Sánchez, et al., 2016; Forsyth, 1980; Forsyth, O'boyle, y McDaniel, 2008). Se puede ver el sistema de nodos ramificados que ha sido usado para categorizar los textos en la figura 2:

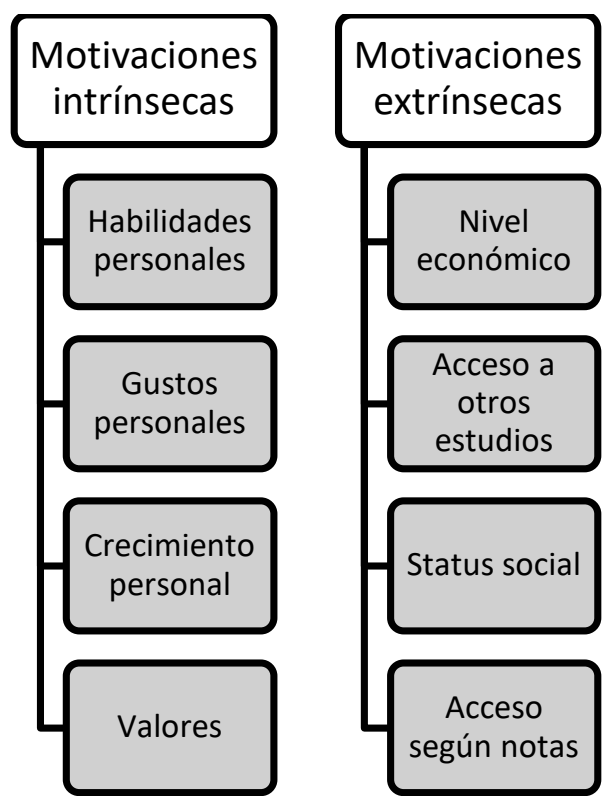

Figura 2: Red de nodos para categorizar textos del alumnado

En función de esa red de nodos, hemos procedido a la categorización de fragmentos de los textos, asociándolos a uno de los tipos generales de motivación y a alguno de los subtipos:

Veamos a continuación algunos ejemplos de categorización de fragmentos de las redacciones del alumnado, atendiendo a los tipos y subtipos de motivación del esquema de nodos utilizado:

Ejemplo 1: Sujetos que quieren estudiar diseño gráfico y física aluden a un criterio de motivación interna formulado como "gusto personal" ${ }^{1}$. 
Sujeto 1: "Me gustaría estudiar esta carrera porque me gustan tanto el dibujo como la informática y además si se trata de diseñar podré usar mi imaginación cosa que me encanta hacer y hago a menudo.

Se me dan muy bien los números, me gustan los problemas y no me cuesta ningún trabajo entenderlos. $Y$ también porque en un futuro me veo de profesora de ESO y Bachillerato, explicando cosas difíciles a niños que tienen interés en aprenderlas, como la física, que se elige si te gusta, porque me gusta tratar con la gente y me amargaría muchísimo yo sola en un despacho o haciendo lo mismo cada día en una oficina, también me gusta la vida que tienen los colegios, me parece muy divertida y la educación un derecho que hay que hacerle ver a los niños, en vez de una obligación o un castigo y eso depende de las ganas y la vocación que tenga un profesor por su trabajo."

Ejemplo 2: Sujeto que quiere estudiar fisioterapia y alude al criterio de motivación extrínseca que hemos categorizado como "nivel económico al que se aspira":

Sujeto 4: "Es una profesión con la que puedes conseguir un buen nivel de vida, debido a que no hace falta tener una consulta para poder practicarla, y puedes empezar trabajando para otros y acabar teniendo tu propia clínica".

Ejemplo 3: Sujeto que refiere en una misma frase argumentos de motivación intrínseca, como el "gusto personal" y extrínseca como el "nivel económico al que se aspira":

Sujeto 7: Me gustaría estudiar ingeniería aerodinámica. Me gustaría estudiar esta carrera porque está relacionada con el mundo de los coches, y es una pasión que viene de mi familia paterna. Es una carrera que está bien pagada y tiene muchas salidas tanto en España como en el extranjero.

Una vez que se ha llevado a cabo el proceso de categorización, el software nos permite obtener la información cruzada para responder a las cuestiones planteadas en los objetivos de investigación. Se presentan a continuación en los resultados de la investigación.

\section{RESULTADOS Y DISCUSIÓN}

De entre los parámetros que podríamos entrar a valorar, como hemos visto en la bibliografía revisada (Rodríguez, Torío, y Fernández, 2006; Navarro y Casero, 2012), este estudio se centra en las variables de motivación intrínseca y extrínseca, dada la importancia que tienen en las posibilidades de incidir en el proceso de motivación.

Siguiendo los objetivos planteados en esta investigación, hemos llevado a cabo una serie de análisis sobre los textos de respuesta de la muestra:

- $\quad$ En primer lugar, se ha examinado la frecuencia de aparición de criterios de motivación intrínseca y extrínseca en toda la muestra, y se observa que, en líneas generales, los criterios de motivación intrínseca (58\%) prevalecen sobre los extrínsecos (42\%), como se ve en la figura 3. 


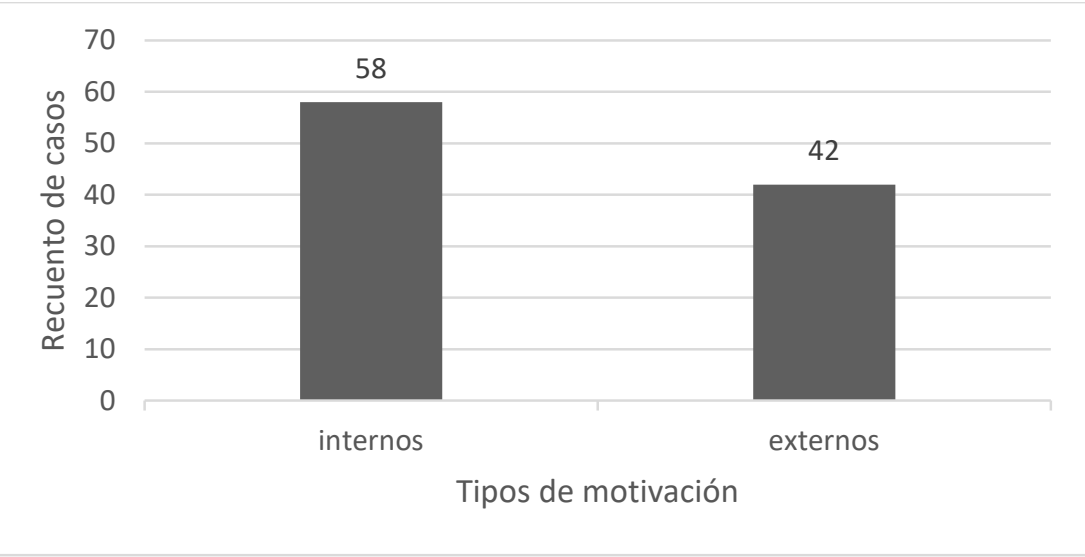

Figura 3: Tipología de criterios de motivación en términos generales según los datos analizados en WebQDA.

A diferencia de otros estudios consultados (Álvarez y Albuerne, 2001), encontramos algunas particularidades de distinción entre modalidades de estudio, en referencia a los criterios de motivación usados en este análisis. Estas frecuencias corresponden, en líneas generales, con los resultados de investigaciones de esta misma temática, llevadas a cabo con anterioridad (Corrales, Sánchez y Moreno, 2016).

- $\quad$ En el siguiente paso se ha analizado la comparación de la importancia que tienen unos y otros criterios en el alumnado de Ciencias y en la modalidad de Humanidades y Ciencias Sociales. Los resultados observados permiten afirmar que el alumnado de Ciencias y Tecnología muestra una menor diferencia entre la cantidad de veces que menciona criterios de motivación intrínseca y extrínseca que el alumnado de Humanidades y Ciencias sociales. Ambos grupos mencionan más abundantemente criterios de motivación intrínseca, pero en el grupo de Humanidades y Ciencias sociales se observa mayor diferencia entre la frecuencia de ambos tipos de criterios. Se puede afirmar que la tendencia se mantiene, aunque con algunos matices, según se aprecia en la figura 4, en la misma línea que vemos en otros estudios revisados (Corrales, Sánchez y Moreno, 2016; Corrales, et al, 2018), aunque la metodología híbrida utilizada en esos estudios permite un contraste de datos cuantitativos con los cualitativos, que dan un mayor equilibrio a los resultados finales.

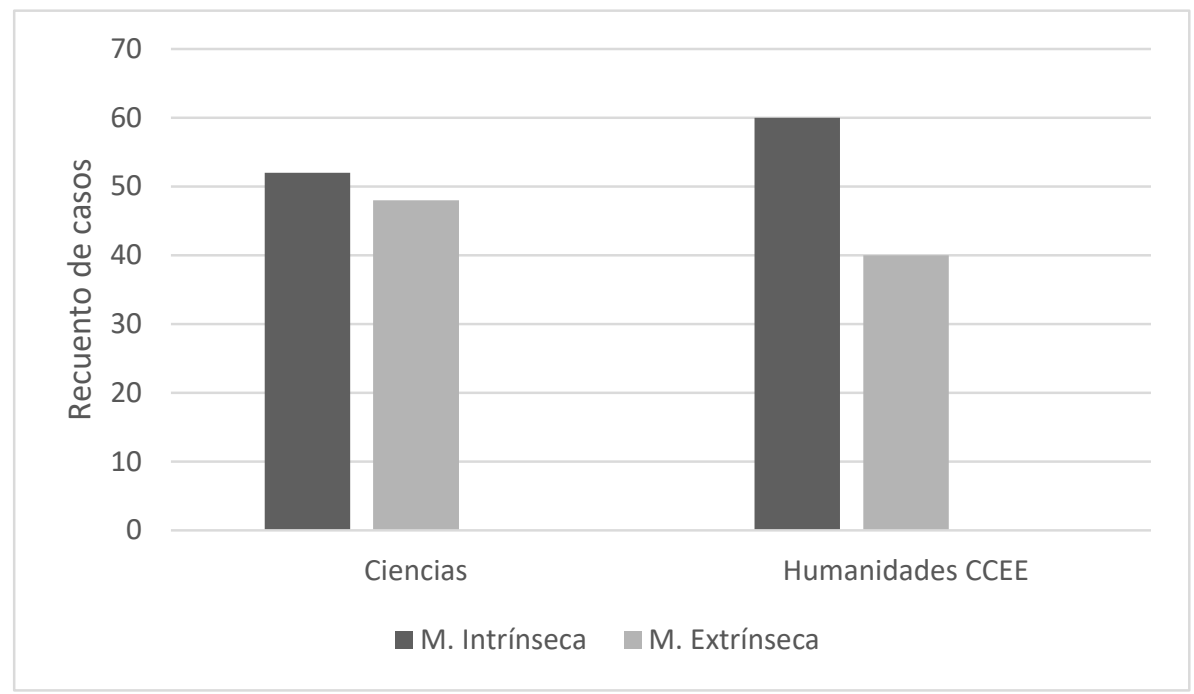

Figura 4: Frecuencia de criterios de motivación por modalidad de estudios 
- $\quad$ En el tercer paso de análisis de los textos aportados por la muestra se entra a valorar la frecuencia de aparición de los diferentes subtipos de motivación intrínseca y extrínseca, se puede encontrar cierto paralelismo con los datos que se han mostrado anteriormente. El alumnado de Bachillerato, en general, parece mostrarse bastante motivado a nivel intrínseco en relación con los gustos personales y a las habilidades que se le dan bien a nivel individual, como se observa en la figura 5. En lo que se refiere a criterios extrínsecos, vemos una mayor incidencia de "nivel económico" y "Estatus social" en el alumnado de Ciencias y Tecnología, aunque ambos grupos los valoran como importantes, mientras que en el alumnado de Humanidades y Ciencias Sociales inciden más que en el Ciencias los factores de acceso a otra titulación o de condicionamiento por la nota, como se muestra en la figura 6.

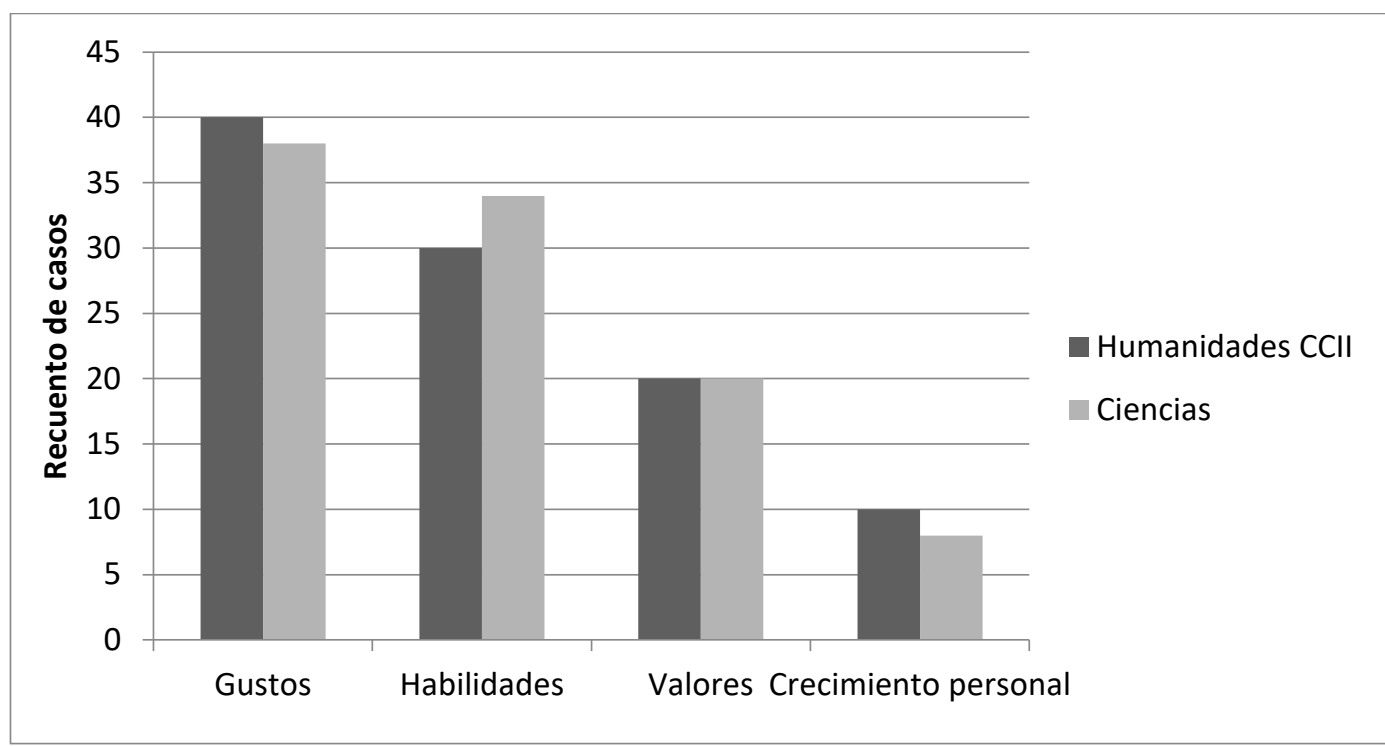

Figura 5: Relación de subtipos de factores de motivación imtrínseca en el alumnado de Ciencias y de Humanidades y Cienccias Sociales.

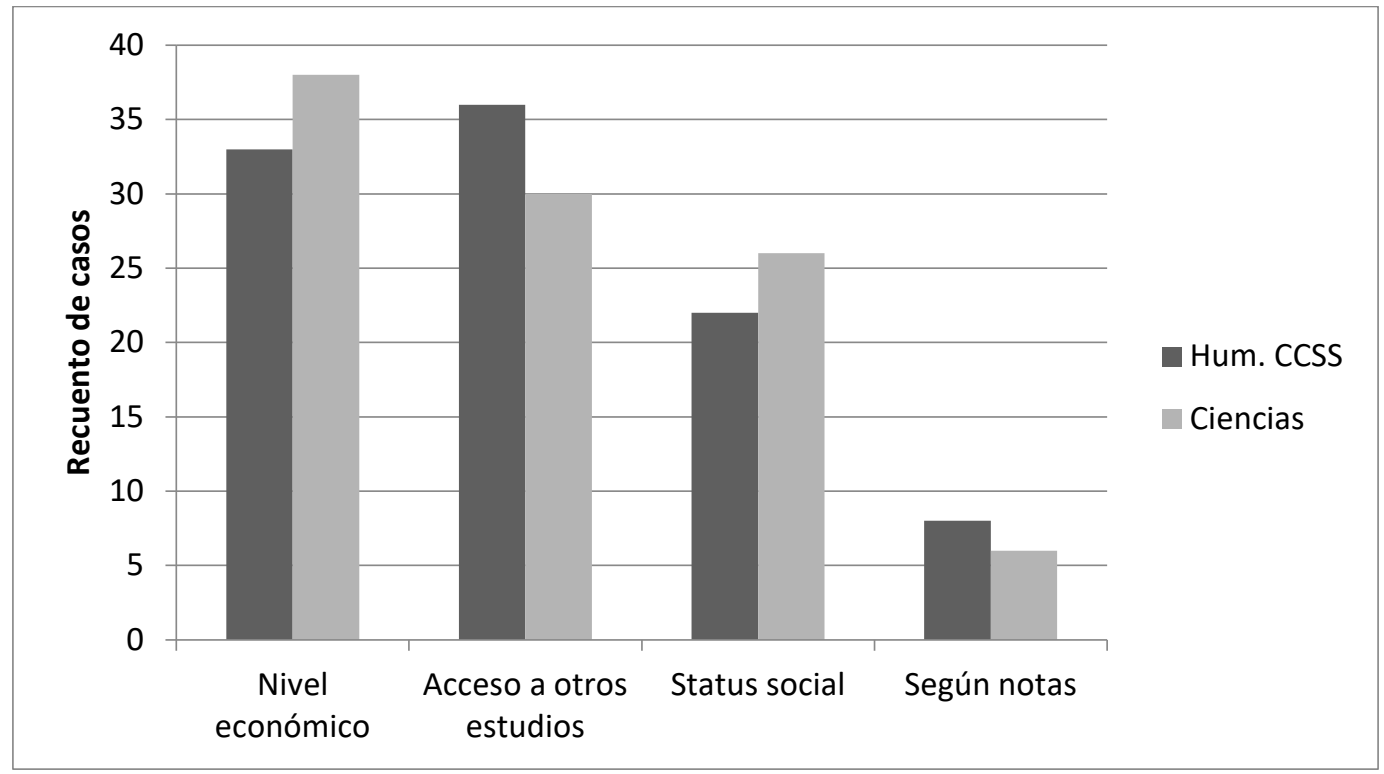

Figura 6: Relación de subtipos de factores de motivación extrínsca en el alumnado de Ciencias y de Humanidades y Ciencias Sociales. 
En relación con esta cuestión, (Moreno, Sanchez y Zamora, 2014) señalan como factores a tener en cuenta la influencia de los otros en la decisión de elegir estudios y la relación entre estudio y ciudadanía. Los participantes en la muestra de nuestro estudio no parecen darle mucho peso a la nota media del curso, en contraste con otros estudios consultados (Martínez, et al, 2015). Son otros elementos, como la posibilidad de acceder a un nivel socioeconómico adecuado o la posibilidad de acceder a una titulación universitaria deseada los factores que más incidencia tienen en sus respuestas.

\section{CONCLUSIONES}

El análisis de resultados que se ha llevado a cabo con los materiales recopilados en el trabajo que se ha realizado con la muestra permiten obtener una serie de conclusiones en relación con los objetivos de investigación de nuestro trabajo. Los objetivos planteados están dirigidos a obtener información acerca de los criterios de motivación intrínsecos y extrínsecos que influyen en la elección de modalidad de estudios del Bachillerato (Cf. Figura 2). A continuación, exponemos estas conclusiones sobre los objetivos planteados:

Objetivo 1: Analizar si es posible detectar motivaciones concretas en el relato de alumnos de Bachillerato sobre su elección de modalidad de estudios.

En los textos de respuestas del alumnado hemos podido detectar expresiones que han podido ser categorizadas como diferentes motivaciones. Es cierto que no todos los factores aparecen con la misma fuerza, pero el esfuerzo de tener que formular factores de motivación para justificar la elección de modalidad de estudio genera la aparición de criterios de motivación en los textos del alumnado de la muestra, que son interesantes para los objetivos de nuestro trabajo.

Objetivo 2: Distinguir elementos de tipo intrínseco y extrínseco en las posibles motivaciones que formule el alumnado de Bachillerato sobre su elección de modalidad.

En primer lugar, se han asociado los párrafos que correspondían a uno de los dos tipos de motivaciones (intrínseca y extrínseca), y después se han relacionado con los diferentes subtipos que se especificaban en la categorización que hemos llevado a cabo. Llama la atención en algunos textos (Ej 3) la naturalidad con la que el alumnado de la muestra fusiona criterios de motivación intrínseca y extrínseca para justificar su elección de modalidad.

Objetivo 3: Establecer distinciones de motivaciones de tipo intrínseco y extrínseco en relación con el alumnado que opta por la modalidad de Ciencias Sociales frente al alumnado de otras modalidades.

Los resultados de la muestra analizada no son demasiado concluyentes en este sentido. Encontramos algunos matices, especialmente en la valoración que el alumnado hace de las motivaciones extrínsecas, que parecen estar presente de modo más claro en el alumnado de Ciencias, aunque los datos no nos permiten extraer conclusiones determinantes.

Este análisis de consecución de objetivos permite obtener las siguientes conclusiones:

- De todo lo expuesto hasta el momento, se concluye el hecho de que se puede detectar la presencia de motivaciones intrínsecas y extrínsecas en el alumnado de Bachillerato para la elección de modalidad de estudios.

- $\quad$ Además, la lectura de datos que se ha llevado a cabo permite afirmar que en la decisión por la modalidad de estudios influyen directamente factores de ambos tipos en alumnado de las modalidades de estudio analizadas. 
- La observación de la frecuencia con la que aparecen los subtipos de motivaciones de tipo extrínseco, muestra una leve diferencia entre las diversas modalidades de estudio: el alumnado de Ciencias y Tecnología menciona de manera ligeramente más reiterada criterios de motivación extrínseca que el alumnado de Humanidades y Ciencias Sociales. Asimismo, hay una mayor oscilación entre la frecuencia con la que el alumnado de Humanidades y Ciencias Sociales menciona criterios de uno y otro tipo, mientras en el grupo de Ciencia y Tecnología, los datos son más parejos.

- La información obtenida resulta de gran utilidad para el proceso de orientación del alumnado en esta etapa de estudios, así como para la implementación de estrategias didácticas motivadoras.

\section{REFERENCIAS}

Álvarez, C. y Albuerne, R. (2001). Rendimiento académico y estilos de aprendizaje en alumnos de segundo de Bachillerato LOGSE. Aula Abierta, 77-85

Atkinson, J. (1983). Personality, motivation, and action: Selected papers. New York: Praeger Publishers.

Ávila, R., Rivero, M. y Domínguez, S. (2010). Metodología de investigación en Didáctica de las Ciencias Sociales. Madrid: (Institución Fernando el Católico, Ed.).

Bacigalupe, M. y Mancini, V. (2012). Motivación y aprendizaje: contribuciones de las neurociencias a la fundamentación de las intervenciones educacionales. Didac, (59), 44-49.

Bandura, A. (1986). Social foundations of thought and action. New Jersey: Prentice Hall.

Bisquerra, R. (2004). Metodología de la investigación educativa (Vol. 1). Madrid: Editorial La Muralla.

Corrales, M., Sánchez, J. y Moreno, J. (2016). The motivation in the teaching of social sciences at the hihg school. NOVA, Journal of education Rechearsh, Vol: 10/2, 175-184.

Corrales, M., Sánchez, J., Moreno, J. y Zamora, F. (2018). Las motivaciones de los jóvenes para el estudio: raíces y consecuencias. Cuadernos de Investigación en Juventud, (4), 60-79.

Cortina, A. (1986). Ética Mınima: introducción a la filosofía práctica. Madrid: Tecnos.

Cortina, A. (2012). Neuroética y neuropolítica: sugerencias para la educación moral. Madrid: Tecnos.

Eisner, E. (1998). El ojo ilustrado. Indagación cualitativa y mejora de la práctica de la enseñanza. Buenos Aires: Paidós.

Forsyth, D. (1980). A taxonomy of ethical ideologies. Journal of Personality and Social psychology, 39 (1), 175-184.

Forsyth, D., O'boyle, E., y McDaniel, M. (2008). East meets west: A meta-analytic investigation of cultural variations in idealism and relativism. Journal of Business Ethics, 83 (4), 813-833.

Hortal, A. (2002). Ética General de las Profesiones. Bilbao, Desclée De Brouwer.

Huertas, J. (1997). Motivación. Querer aprender. Buenos Aires: Aique.

Mallart, J. (2008). Didáctica de la motivación. Madrid: McGraw Hill.

Marina, J. (2013). Talento, motivación e inteligencia: Las claves para una educación eficaz. Barcelona: Grupo Planeta (GBS).

Martínez, A., Sánchez, M., Ortega, F. y Zurita, M. (2015). La elección de estudios superiores universitarios en función de la modalidad de estudios, la nota media y el género. Magister, 27(1), 18-25.

Maslow, A. (1991). Motivación y personalidad. Madrid: Ediciones Díaz de Santos.

Moreno, J., Sánchez J., Zamora, F. (2014). La preocupación por la ética en la universidad. Fundamentos teóricos y aplicaciones prácticas». En Ética en las aulas. Desarrollo de prácticas docentes éticamente responsables. Una aproximación experimental desde la Universidad de Extremadura. Madrid: Entimema.

Moreno, J. (2015). Estudio "con-sentido" en la Universidad. Motivación del estudio en los universitarios extremeños y el bien interno de las profesiones. Badajoz: Universidad de Extremadura

Navarro,C., y Casero, A. (2012). Análisis de las diferencias de género en la elección de estudios universitarios. Estudios sobre educación, 22, 115-132.

Pintrich, P. Schunk, D. y Luque, M. (2006). Motivación en contextos educativos: teoría, investigación y aplicaciones. Barcelona: Pearson Prentice Hall.

Quecedo, R., y Castaño, C. (2002). Introducción a la metodología de investigación cualitativa. Revista de psicodidáctica, (14).

Queija, I., Jiménez, V., Ramírez, P., Flores, M., Gallardo, M.., Vega, M. y Alamillo, R. (2014). Manual de Psicología de la Educación. Ediciones Pirámide.

RAE. (2014). Diccionario de la lengua española. Planeta Publishing.

Rodríguez, M., Torío, S. y Fernández, C. (2006). El impacto del género en las elecciones académicas de los estudiantes asturianos que finalizan la ESO. Revista española de orientación y psicopedagogía, 17 (2). 13-18.

Sánchez, J., Zamora F., Alías D., Moreno, J. y Espejo, L. (2016). ¿Ética en la educación? Instrumentos para medir, potenciar y desarrollar el razonamiento moral en ámbitos educativos multinivel. En Libro de actas CIMIE16 de AMIE. Recuperado a partir de http://amieedu.org/actascimie16.

Sandín, M. (2003). Investigación cualitativa en educación. Fundamentos y tradiciones. Madrid: Mc Graw and Hill Interamericana.

Souza, F., Costa, A., Moreira, A., Souza, D., y Freitas, F. (2016). WebQDA: manual de utilização rápida. Aveiro: UA Editora.

Weiner, B. (1985). An attributional theory of achievement motivation and emotion. Psychological review, 92 (4), 548563.

Woolfolk, A. (2006). Psicología educativa. Madrid: Pearson educación.

${ }^{1}$ La numeración de sujetos corresponde al proyecto elaborado en WebQDA para el desarrollo de la investigación. 